\title{
BMJ Open Disparities in the completion of steps to kidney transplantation: protocol for a systematic review
}

\author{
Heather M Traino, ${ }^{1}$ Camilla W Nonterah, ${ }^{2}$ John W Cyrus, ${ }^{3}$ Avrum Gillespie, ${ }^{4}$ \\ Megan Urbanski, ${ }^{1}$ Michael Adair-Kriz ${ }^{5}$
}

To cite: Traino HM, Nonterah CW, Cyrus JW, et al. Disparities in the completion of steps to kidney transplantation: protocol for a systematic review. BMJ Open 2015;5:e008677. doi:10.1136/bmjopen-2015008677

- Prepublication history and additional material is available. To view please visit the journal (http://dx.doi.org/ 10.1136/bmjopen-2015008677).

Received 4 May 2015 Revised 19 August 2015 Accepted 21 August 2015

CrossMark

For numbered affiliations see end of article.

Correspondence to Dr Heather M Traino; trainohm@temple.edu

\section{ABSTRACT}

Introduction: Disparities in access to transplantation have been well documented. The extant literature, however, focuses largely on disparities and related barriers for African-American patients and none has used the steps to transplantation as a guiding framework. This review will catalogue disparities in the steps to transplantation as well as the barriers and facilitators to completion of each step identified in the extant literature. The results of the review will be used to generate recommendations for future research to improve equity in access to kidney transplantation.

Methods and analysis: Standard procedures will be used in the conduct of the review. Searches will be performed using the following electronic databases: PubMed/Medline, PsycINFO, CINHAL, EMBASE, Cochrane library and Web of Science. Reports of original research will be eligible for inclusion if they are published from 2005 to present, written or available in English language, performed in the USA, enrol adult participants (18 years of age or more), and employ descriptive or observational designs. Two authors will independently screen retrieved articles for inclusion. MaxQDA will be used for data analysis and management. All included reports will be coded for article characteristics; disparities identified; barriers and motivators of completion of steps to transplantation; and proposed solutions to disparities and barriers. Each report will be coded independently by two authors and discrepancies resolved by discussion among the full team. A qualitative approach to data analysis is planned. Risk of bias will be assessed using standard procedures.

Ethics and dissemination: The findings will provide crucial information on the current status of disparities in access to transplantation. PRISMA guidelines will be followed in reporting the results of the review. It is anticipated that these results will inform research which seeks to increase parity in access to transplantation.

Systematic review registration: PROSPERO CRD42014015027.

\section{INTRODUCTION}

By recent estimates, over 20 million Americans suffer from chronic kidney disease, of whom

\section{Strengths and limitations of this study}

- This article describes the protocol for a systematic review of articles published from 2005 to present on disparities in access to kidney transplantation.

- The review will be conducted using standard procedures and reported per the Preferred Reporting Items for Systematic Reviews and Meta-Analysis (PRISMA) guidelines.

- As with any systematic review there is the potential for the results to be skewed due to reporting bias.

approximately 637000 are diagnosed with end-stage renal disease (ESRD). ${ }^{1}$ Gender and ethnic/racial differences in the development and treatment of ESRD are well documented. ${ }^{1}$ For instance, the incidence rate is $50 \%$ higher for men as compared to women, and African-Americans and Native Americans are 3.3 and 1.5 times more likely to develop ESRD than non-Hispanic Whites. ${ }^{1}$ Additionally, although kidney transplantation, particularly living donor transplants, offers the best chance for patient survival, the likelihood of receiving a transplant decreases with age, female sex and for ethnic minorities. ${ }^{1}$ According to the Organ Procurement and Transplantation Network, of the 17105 kidney transplants performed in 2014, only 4316 (25.2\%) and 2738 $(16.0 \%)$ were performed in AfricanAmericans and Hispanics, respectively, despite the high prevalence of ESRD in these populations. $^{2}$

Disparities, or unfair and preventable differences, ${ }^{3}{ }^{4}$ in access to transplantation have been identified as well. Indeed, potentially avoidable barriers have been documented in the determination of suitability for referral to a transplant centre; interest in transplantation; receipt of a referral to a transplant centre for evaluation; completion of the initial visit to a transplant centre; completion 
of the evaluation for transplantation; successful transplant candidacy; identification of a living donor; and, receipt of a living or deceased donor transplant. ${ }^{5-9}$ Racial minorities, women, older patients and the poor are among those most likely to experience barriers along the road to transplantation. ${ }^{5}$

Previous reviews have implicated a combination of factors including income, comorbidities, insurance issues and social support, for these disparities. ${ }^{710}$ These studies, however, are outdated and focus largely on disparities and barriers for African-American patients only. Moreover, none of the reviews conducted to date use the steps to transplantation as framework for categorising identified disparities. This review builds on these previous efforts and is guided by the following research question (RQ):

RQ1: What is known about disparities in the steps to kidney transplantation?

The findings of the review will be used to generate recommendations for future research needed to reduce disparities and increase parity in access to kidney transplantation.

\section{METHODS AND ANALYSIS}

To ensure the review is of high caliber and to minimise bias, standard procedures for the conduct of a systematic review will be utilized; ${ }^{11}{ }^{12}$ we will adhere to the Preferred Reporting Items for Systematic Review and Meta-Analysis (PRISMA) guidelines in reporting the results of the review. ${ }^{13}{ }^{14}$ Protocol amendments will be documented by date and rationale, and published with the results of the review.

\section{Eligibility criteria}

Studies eligible for inclusion will be written or available in English language, performed in the USA, enrol adult participants (18 years of age or above), and employ descriptive or observational designs (eg, cross-sectional, cohort, etc). Reports of original research examining any of the following steps to kidney transplantation will be included in the review: interest in transplantation; referral to transplant centre; first visit to transplant centre; completion of the work-up or evaluation for transplantation; waitlisting for transplant; receipt of living or deceased donor kidney transplantation, including preemptive transplantation. ${ }^{15}$ Reports of research examining healthcare providers' attitudes and perspectives or the pursuit of live donor kidney transplantation will be excluded since systematic reviews have been published on these topics. ${ }^{78}$ Studies examining other solid organ types, such as heart and liver, as well as studies with child or adolescent samples will be excluded from the review. Editorial papers, comments, personal viewpoints and previous literature reviews will also be excluded.

\section{Information sources}

Databases which cover disciplines in the life sciences, social sciences, biomedical topics, nursing and healthcare will be searched. Specifically, these searches will be performed using electronic databases; PubMed/ Medline, EMBASE, PsycINFO, CINAHL, Web of Science and the Cochrane Central Register of Controlled Trials. Reference lists of previously published reviews and studies meeting the inclusion criteria will be searched for other relevant citations. We will also search leading transplant journals (eg, American Journal of Transplantation) for published abstracts. Our search for grey literature will include online collections of dissertations and theses as well as individual requests to researchers and experts in the fields of transplantation and health disparities for unpublished manuscripts. The review will include studies from 2005 to present.

\section{Search strategy}

A research librarian with experience in the conduct of systematic reviews (JWC) will lead the development and refinement of the search strategy. An iterative process will be employed in the search such that, as relevant research is identified and retrieved and new search terms added, the search strategy will be modified and the search started anew. This will ensure that the full range of relevant research reports is captured in the search process. The final search strategy will be documented, with the number of retrieved articles (ie, hits) at each stage, and tested for replicability before ending the search process. The strategy will be modified as appropriate for use in each database searched. A draft of the strategy for use in PubMed is provided in the online appendix.

\section{Data management}

Articles retrieved in the search process will be imported into RefWorks, reference manager software, for further review for eligibility; Microsoft Excel will be used for tracking search results (eg, electronic searches, hand searches) and article disposition (included/excluded). Eligible articles will be imported into MaxQDA, software developed to facilitate project management and qualitative analyses, along with the coding scheme and codes (described below).

\section{Selection process}

Two members of the study team (CWN and HMT) will independently perform the initial screening of titles and abstracts of studies retrieved and saved to RefWorks to separate irrelevant literature from the body of work to be further assessed for eligibility. Agreement between screeners' determination of articles to be excluded will be assessed and disagreements discussed to achieve consensus. The remaining articles will be assessed independently for inclusion by two members of the research team; disagreements will be discussed by the full team until a final disposition is reached. Following PRISMA guidelines, a flowchart will be created to provide a visual representation of the studies included and excluded from the review, with explanations provided for those excluded. ${ }^{13}$ 


\section{Data collection process}

A coding schema and corresponding protocol have been developed for data extraction. An iterative process was employed to develop the schema, with multiple rounds of revisions and pilot testing. The final coding form, including all codes, will be provided on request to the first author $(\mathrm{CWN})$. The following data will be extracted from each article: publication characteristics (ie, year of publication, author names and affiliation, and funding source); study characteristics (ie, study design, purpose, theory used, type of disparity discussed, measures employed, and statistical analyses) and, when appropriate, characteristics of the intervention (ie, description of the intervention, type of comparison group, blinding); sample characteristics (ie, sample size and calculation, and description of the sample); barriers and motivators to patients' completion of the steps to transplantation, including proposed solutions to overcome barriers and/or remedy disparities; and, study results, including study limitations and threats to validity. The final code system will be added to MaxQDA for data analysis. Each included article will be randomly assigned to and coded independently by two members of the research team using MaxQDA.

\section{Outcomes}

Outcomes of the review include known disparities in the completion of each of the eight steps to transplantation as well as the barriers and motivators to the completion of each step among disparate groups.

\section{Assessment of bias}

Each study will be critically assessed for risk of bias using the Mixed Methods Appraisal Tool, which has provided reliable quality scores for qualitative, quantitative and mixed methods studies. ${ }^{16}$ Further, we will identify study limitations or threats to validity as an additional means of assessing the quality of each study included in the review. Experimental studies testing interventions will be evaluated using the Cochrane Risk of Bias tool. ${ }^{17}$

\section{Data analysis}

We will take a qualitative approach to data analyses. Specifically, the data will be synthesised narratively based on the codes and themes found in the research reports included in the review. A general description of the sample of articles included in the review, including frequency counts and percentages for articles focusing on each of the eight steps to transplantation and data collection methods employed, will be provided in a table and summarised in text. Additionally, we will tabulate identified disparities by type (eg, racial/ethnic, sex, age, etc) and step to transplant and the associated barriers and facilitators of progress along the path to kidney transplantation. Any missing data from reviewed articles will be sought through direct contact with the authors. The impact of missing data on the study findings will be discussed.

\section{Meta-biases}

We will attempt to reduce the likelihood of reporting biases through the inclusion of unpublished and grey literature in the review. The Newcastle Ottawa Scale will be used to assess the risk of bias in reviewed studies employing nonrandomised designs. ${ }^{18}$ For randomised trials, we will use funnel plots to detect and assess the magnitude of reporting biases. ${ }^{19}$ We will also use the 11-item Assess the Methodology Quality of Systematic Reviews (AMSTAR) tool to examine the methodological quality of the review. ${ }^{20} 21$

\section{ETHICS AND DISSEMINATION}

The results of this review will catalogue disparities at each step of the transplant process and assess the impact of interventions aimed at overcoming barriers to transplantation, with the goal of recommending strategies for reducing or eliminating disparities in the transplant process. As with any type of research, certain limitations are anticipated. It is likely that the studies included in the review lack homogeneity, limiting our ability to perform a meta-analysis. It may be difficult to obtain access to unpublished data since authors may be unaware of the various sources of data related to the review topic. However, authors will assess for publication bias in an attempt to reduce this potential limitation.

We anticipate that the findings of this review will be of interest to multiple stakeholders, including healthcare professionals working the field of transplantation and responsible for the care of patients in need of transplants, hospital leadership and administration, researchers and policymakers. As such, our dissemination plan includes publication in academic journals as well as presentations at relevant conferences, symposia and professional meetings.

As this is a systematic review of completed and/or published studies, ethics approval is not required.

\section{PROTOCOL REGISTRATION}

This protocol has been registered with the PROSPERO International Prospective Register of Systematic Reviews (registration number: CRD42014015027).

Author affiliations

${ }^{1}$ Department of Social \& Behavioral Sciences, Temple University, Philadelphia, Pennsylvania, USA

${ }^{2}$ Department of Psychology, Virginia Commonwealth University, Richmond, Virginia, USA

${ }^{3}$ Tomkins-McCaw Library Virginia Commonwealth University, Richmond, Virginia, USA

${ }^{4}$ Department of Medicine Section of Nephrology Hypertension and Kidney Transplantation, Temple University School of Medicine, Philadelphia, Pennsylvania, USA

${ }^{5}$ Department of Public Health, Temple University, Philadelphia, Pennsylvania, USA

Contributors CWN, HMT conceptualised the review, planned the protocol and serve as guarantors; AG provided clinical expertise and advice on the protocol; JWC devised the search strategy; JWC, CWN, HMT will search for and screen studies for potential inclusion in the review; CWN, AG, MU, HMT, MA-K will extract and analyse the data. All authors participated equally in drafting and revising the protocol for publication. 
Funding This project is supported in part by Grant number K01HS018113 from the Agency for Healthcare Research and Quality (AHRQ). The content is solely the responsibility of the authors.

Competing interests None declared.

Provenance and peer review Not commissioned; externally peer reviewed.

Open Access This is an Open Access article distributed in accordance with the Creative Commons Attribution Non Commercial (CC BY-NC 4.0) license, which permits others to distribute, remix, adapt, build upon this work noncommercially, and license their derivative works on different terms, provided the original work is properly cited and the use is non-commercial. See: http:// creativecommons.org/licenses/by-nc/4.0/

\section{REFERENCES}

1. National Institutes of Health. National Institute of Diabetes and Digestive and Kidney Diseases, Bethesda, MD 2014. United States Renal Data System, 2014 USRDS Annual Data Report: an Overview of the epidemiology of kidney disease in the United States. http:// www.usrds.org/2014/view/v2_01.aspx

2. Organ Procurement and Transplantation Network [Internet]. 2015 [cited 6 May 2015]. http://optn.transplant.hrsa.gov

3. Ladin K, Rodrigue JR, Hanto DW. Framing disparities along the continuum of care from chronic kidney disease to transplantation: Barriers and interventions. Am J Transplant 2009;9:669-74.

4. Whitehead M. The concepts and principles of equity and health. Int $J$ Heal Serv 1992;22:429-45.

5. Alexander GC, Sehgal AR. Barriers to cadaveric renal transplantation among blacks, women, and the poor. JAMA 2015;280:1148-52.

6. Lunsford SL, Simpson KS, Chavin KD, et al. Can family attributes explain the racial disparity in living kidney donation? Transplant Proc 2007;39:1376-80.

7. Navaneethan SD, Singh S. A systematic review of barriers in access to renal transplantation among African Americans in the United States. Clin Transplant 2006;20:769-75.
8. Purnell T, Hall Y, Boulware L. Understanding and overcoming barriers to living kidney donation among United States racial and ethnic minorities. Adv Chronic Kidney Dis 2012;19:244-51.

9. Weng FL, Joffe MM, Feldman $\mathrm{HI}$, et al. Rates of completion of the medical evaluation for renal transplantation. Am J Kidney Dis 2005;46:734-45.

10. Churak JM. Racial and ethnic disparites in renal transplantation. J Natl Med Assoc 2005;97:153-60.

11. Higgins J, Green S, eds. Cochrane handbook for systematic reviews of interventions. Chichester, UK: John Wiley \& Sons, 2008.

12. Petticrew M, Roberts $H$. Systematic reviews in the social sciences: a practical guide. Malden, MA: Blackwell, 2006.

13. Moher D, Liberati A, Tetzlaff J, et al. Preferred reporting items for systematic reviews and meta-analyses: the PRISMA statement. BMJ 2009;339:b2535.

14. Moher D, Shamseer L, Clarke M, et al. Preferred reporting items for systematic review and meta-analysis protocols (PRISMA-P) 2015 statement. Syst Rev 2015;4:1.

15. Sullivan C, Leon JB, Sayre SS, et al. Impact of navigators on completion of steps in the kidney transplant process: a randomized, controlled trial. Clin J Am Soc Nephrol 2012;7:1639-45.

16. Pace R, Pluye P, Bartlett G, et al. International Journal of Nursing Studies Testing the reliability and efficiency of the pilot Mixed Methods Appraisal Tool (MMAT) for systematic mixed studies review. Int J Nurs Stud 2012;49:47-53.

17. Higgins $J$, Altman D. Assessing risk of bias in included studies. In: Higgins J, Green S, eds. Cochran handbook for systematic reviews of interventions. Chichester, UK: John Wiley \& Sons, 2008:187-242.

18. Wells G, Shea B, O'Connell J, et al. The Newcastle-Ottawa Scale (NOS) for assessing the quality of nonrandomized studies in meta-analyses. PLoS Negl Trop Dis 2013;7:e2195.

19. Sterne J, Egger M, Moher D. Addressing reporting biases. In: Higgins J, Green S, eds. Cochran handbook for systematic reviews of interventions. Chichester, UK: John Wiley \& Sons, 2008:297-334.

20. Shea BJ, Grimshaw JM, Wells GA, et al. Development of AMSTAR: a measurement tool to assess the methodological quality of systematic reviews. BMJ Med Res Meth 2007;7:10.

21. Shea BJ, Hamel C, Wells GA, et al. AMSTAR is a reliable and valid measurement tool to assess the methodological quality of systematic reviews. J Clin Epidemiol 2009;62:1013-20. 